\title{
Analysis of a MIMO-OFDM Wireless Communication System Using a Binary Power Control Scheme with Radio Channel Uncertainties
}

\author{
Santosh M.Tondare*, Veeresh G. Kasabegoudar \\ P. G. Department, MBES College of Engineering, Ambajogai, India \\ *Corresponding author: santoshtondare12@gmail.com
}

Received July 01, 2014; Revised August 02, 2014; Accepted August 07, 2014

\begin{abstract}
Here a novel binary power control scheme is presented with radio channel uncertainty which includes path loss, Rayleigh fading, shadowing coefficient, and interference from other users to analyse the performance of MIMO-OFDM wireless communication system. From bit error rate (BER) point of view a two tier system with adjacent interference is taken into account to guarantee QoS. The main objective of this system is to optimize energy efficiency with QoS. Taking both energy efficiency and quality of service (QoS) an energy efficient binary power control with BER constraint algorithm is presented. The performance of this system is analyzed and results of the same are presented to show the effectiveness of the system presented.
\end{abstract}

Keywords: wireless communication, power control, MIMO-OFDM, bit error rate, quality of service

Cite This Article: Santosh M.Tondare, and Veeresh G. Kasabegoudar, "Analysis of a MIMO-OFDM Wireless Communication System Using a Binary Power Control Scheme with Radio Channel Uncertainties." American Journal of Electrical and Electronic Engineering, vol. 2, no. 4 (2014): 129-142. doi: 10.12691/ajeee-2-4-1.

\section{Introduction}

With rapid development of information and communication technologies (ICT), the energy consumption has grown up to 163 PJ i.e. two billion litres of diesel is used by the tower stations emitting around 32.9 millions tones of $\mathrm{CO}_{2}$ $[1,2]$ and is around $2 \%$ of worldwide $\mathrm{CO}_{2}$ emission. With the recent spectrum reframing from $900 \mathrm{MHz}$ to 1800 $\mathrm{MHz}$ and ongoing 3G, upcoming 4G roll outs, the subscriber base is consistently increasing and is currently more than one billion. This growth will require 1,00,000 more towers to ensure network availability [2]. The rapid increase in number of users is causing burden on network operators from economical perspectives. The main contributing sectors within the ICT industry include the energy requirements of PCs and monitors (40\%), data centres about $23 \%$ and, fixed \& mobile telecommunications contribute about $24 \%$ of the total emissions. A typical communications company spends nearly $1 \%$ of its revenues on energy which for large operators may amount to hundreds of crores of rupees [3]. To overcome these economical and environmental challenges raised by energy consumption, ICTs are moving towards green wireless communication approach. These in terms are shifting towards energy efficient designs in all stages of cellular networks while guaranteeing user's QoS. The green wireless communication explores energy savings of cellular networks in hardware design and manufacture, node deployment, and network operation and management [4,5]. A holistic approach was proposed for component link and network level energy savings in cellular networks [4]. These efforts include reductions in the electricity required to power network elements, integration of renewable energy sources such as solar and wind, more energy efficient practices for network operations and a greater focus on recycling and reuse of network equipment. Since energy consumption is closely related to network utilization and life time [6], the network-level energy efficiency is believed to be one of the promising optimization targets. Furthermore, for most power allocation schemes, the acquisition for the perfect centralized knowledge of channel state information (CSI) is a great challenge [7] to tackle this difficulty; a binary power control (BPC) scheme which leads to a simpler or even distributed solution for performance optimization was proposed [8]. However in many power control algorithms channel gain is considered as a random variable [9] in spite of considering various influencing factors as pass loss, shadowing and fading effect [10]. The detailed theory on this topic can be found in [11,12]. In the proposed algorithm a two tier system with the channel interference is considered. Based on these parameters the channel state information (CSI) is obtained and power allocation is done with either $\mathrm{P}_{\max }$ or $\mathrm{P}_{\min }$.

The system presented in this paper is analysed using MATLAB. Section 2 presents the system model of the proposed wireless system. Binary power control scheme is presented in Section 3. Section 4 presents the performance analysis of entire system and discussions on the measured results are also presented there. Finally, conclusions of this study are presented in Section 5. 


\section{System Model}

Figure 1 shows the model of proposed MIMO-OFDM wireless communication system with base station $\mathrm{B}_{\mathrm{T}}$ located at the center of the cell with a protection distance of $\mathrm{D}$ and first tier mobile unit (receiving $\mathrm{M}_{\mathrm{T}}$ ) with a distance of (D-R) and second tier mobile units with $(D+R)$ distance.

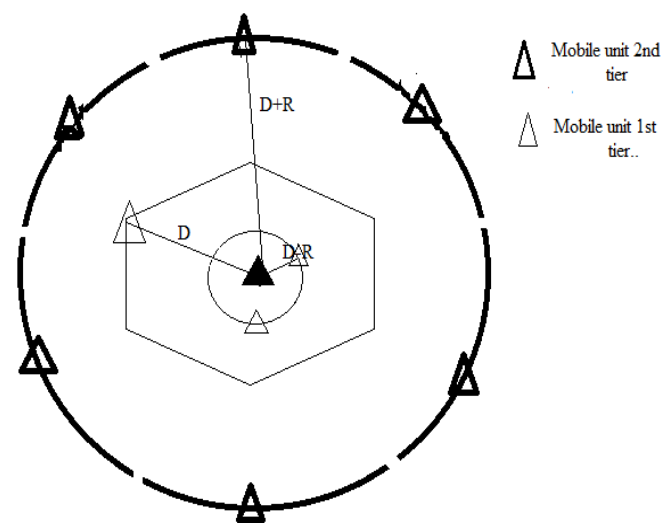

Figure 1. Proposed system model

Each of the signal travelling form transmitting antenna undergoes several radio channel uncertainties like path loss, shadowing coefficient, multipath fading coefficient, and interference from other users. Assuming all these parameters the received signal is given by [13]

$$
S=\frac{w z_{i}^{2}}{R_{i \sigma_{r}}} p_{i}
$$

For first tier mobile unit it is given by

$$
S=\frac{w z_{i}^{2}}{(D-R)_{\sigma_{r}}} p_{i}
$$

For second tier mobile unit it is given by

$$
S=\frac{w z_{i}^{2}}{(D+R)_{\sigma_{r}}} p_{i}
$$

Where $\mathrm{R}$ is the distance between base station and user $\mathrm{i}, \mathrm{z}$ is the Rayleigh fading coefficient, $\mathrm{w}$ is lognormal coefficient, and $\sigma_{r}$ is the path loss coefficient.

Energy efficiency optimization is carried out by defining energy efficiency which is the ratio of channel capacity to the total power transmission with reference to Shannon capacity limit

$$
\begin{gathered}
\eta=\frac{\text { channel capacity }}{\text { total power transmittd }} \\
\eta=\frac{\sum_{i=1}^{N} \log _{2}\left(1+\frac{S_{i}}{n_{o}}\right)}{P_{\text {total }}}
\end{gathered}
$$

Where $S_{i}$ is the received signal and $n_{0}$ is the AWGN in wireless sub-channel.

Here BPSK modulation scheme to investigate BER performance is adopted and is given by [13]

$$
\mathrm{P}_{\mathrm{BER}}=\frac{1}{2} e^{-\frac{\varepsilon_{b}}{N_{O}}}
$$

\section{Binary Power Control Scheme}

Binary power control is a power control scheme with only two allowable power values, usually 0 or $\mathrm{P}_{\max }$. Hence, a link can either transmit at a full power or be switched off completely. Binary power control (BPC) has the advantage of leading towards simpler or even distributed power control algorithms. Moreover, the binary power control scheme is formulated as [14]

$$
\mathrm{P}_{\min } \leq \mathrm{P} \leq \mathrm{P}_{\max }
$$

Assuming this scheme, the number of subchannels are divided into two subsets one with minimum transmission power $\left(\mathrm{MP}_{\min }\right)$ and other with maximum transmission power $\left(\mathrm{MP}_{\max }\right)$. However, total number of channels used are $\mathrm{N}$ and subchannels are grouped into $\mathrm{M}$. The wireless subchannel with maximum power is given by [13]

$$
\mathrm{P}_{\max -1}=\frac{P_{\text {maxtotal }}}{M}
$$

And, energy efficiency is as given in [13],

$$
\eta=\frac{\sum_{i=1}^{M} \log _{2}\left(1+\frac{\frac{w z_{i}^{2}}{R_{i \sigma_{r}}} p_{\text {max_1 }}}{n_{o}}\right)}{P_{\text {maxtotal }}}
$$

\section{Performance Analysis}

Based on the above discussed constraints i.e. firstly the subchannels are ordered digressively according to channel state information (CSI). Then the subchannels will either be allocated with Pmax or Pmin. After this the process of determining BER is carried out. This average bit error rate is compared with the threshold value (b) to guarantee the QoS. Typical values of radio channel uncertainties are listed in Table 1.

Table 1. Different parameters considered in EEBPCB algorithm

\begin{tabular}{|c|l|c|}
\hline Sr.No. & \multicolumn{1}{|c|}{ Parameter } & Typical values \\
\hline 1 & No Of Subchannels & $8-128$ \\
\hline 2 & Total Transmitted Power & $0.6-1.0$ \\
\hline 3 & Bandwidth(Hz) & $1 \mathrm{MHz}$ \\
\hline 4 & Cell radius(m) & $1-500$ \\
\hline 5 & Path loss Coefficient & $3.8-4.1(2-4)$. \\
\hline 6 & Protection Distance $(\mathrm{d})$ & $50 \mathrm{~m}$ \\
\hline 7 & Spectrum Efficiency $\left(\mathrm{n}_{0}\right)$ & $0.1 \mathrm{w}$ \\
\hline
\end{tabular}

From Figure 2 it is observed that for increase in number of subchannels in EEBPCB, the energy efficiency gets increased in comparison with average power control algorithm presented in [15].

Figure 3 shows the energy efficiency comparison of EEBPCB algorithm and average power control algorithm [16] as a function of total transmitted power. Here, as the signal power increases, the energy efficiency of MIMOOFDM system goes on reducing. However, EEBPCB algorithm shows high efficiency for different power ratings in comparison with average power control algorithm [16]. 


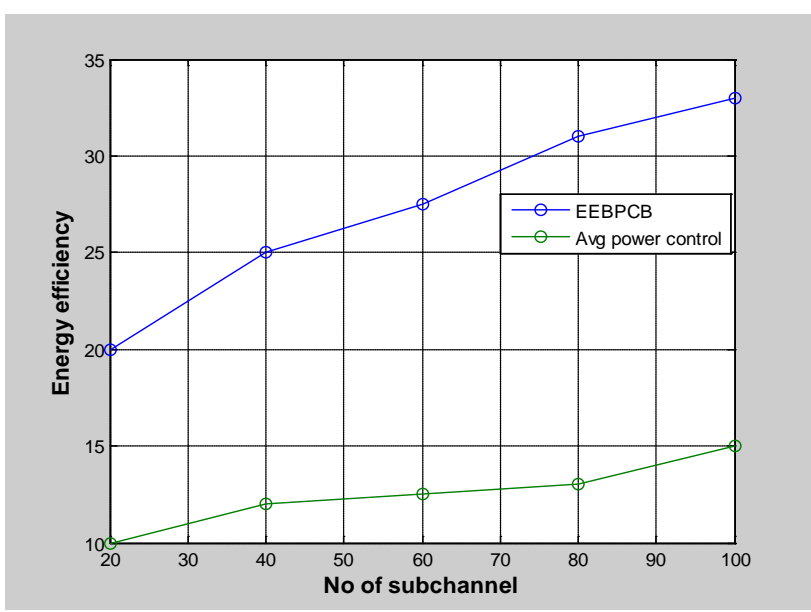

Figure 2. Energy efficiency comparison for EEBPCB and average power control algorithm for different no of subchannels

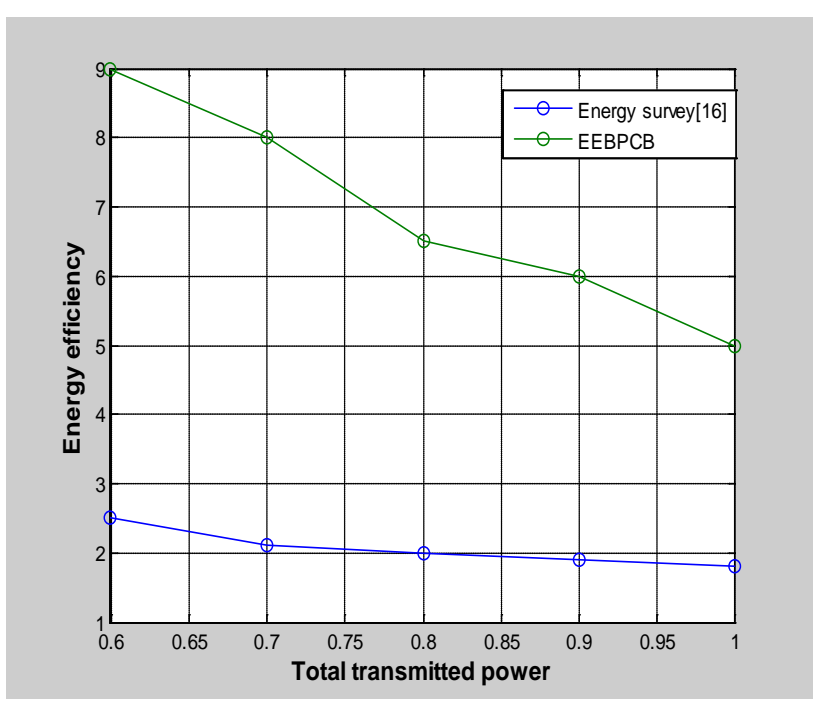

Figure 3. Energy efficiency comparison for EEBPCB and average power control algorithm with different transmitted power

In another effort, bit error rate comparison of EEBPCB and EBPCB [13] as a function of different path loss coefficients is presented in Figure 4. However, for both the schemes the bit error rate is ascending with path loss coefficients. In case of EEBPCB algorithm there is a marginal loss in average bit error rate as compared with EBPCB.

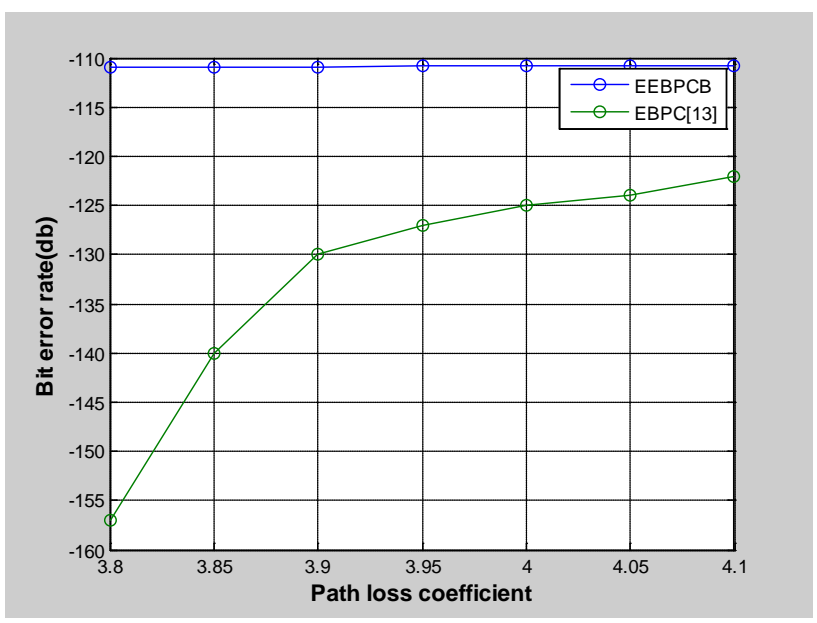

Figure 4. Bit error rate comparison of EЕBPCB and ЕВPCB as a function of different path loss coefficients

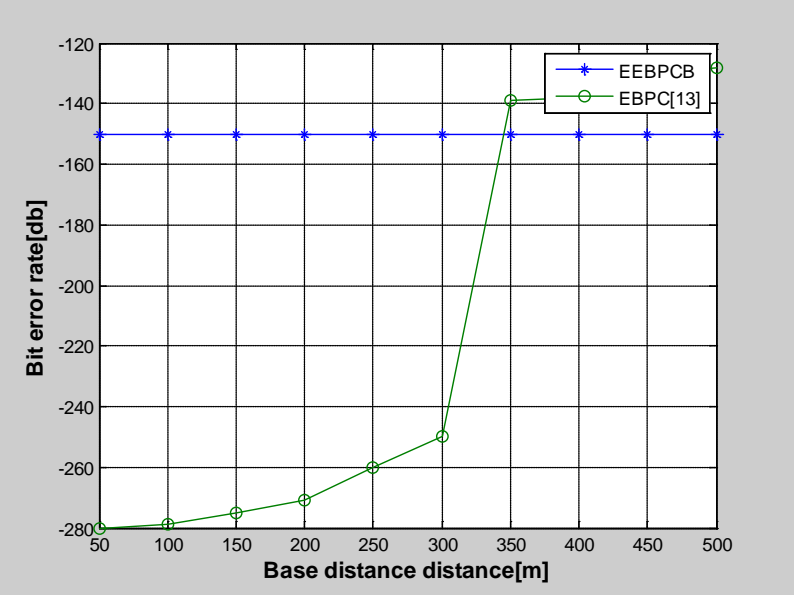

Figure 5. Bit error rate comparison of EEBPCB and EBPCB with distance form base station

Figure 5 shows bit error rate comparison of EEBPCB and EBPCB [13] as a function of distance from base station. In EEBPCB algorithm we have considered different interference scenarios from other users i.e. the distance from the base station is equal to $50 \mathrm{~m}$ (D), greater than $50 \mathrm{~m}(\mathrm{D}+\mathrm{R})$, and less than $50 \mathrm{~m}$ (D-R). From the results presented here it is shown that the BER is nearly constant for all cases considered.

\section{Conclusions}

The performances of MIMO-OFDM wireless communication system considering radio channel uncertainties have been analysed. The exact impact of the proposed system interns of energy efficiency, average bit error rate has been fully investigated under different scenarios like number of channels, total transmission power, distance from the base station, and path loss coefficients. Results presented show that energy efficiency of proposed system model is better than the other techniques mentioned, also the BER is nearly constant for varying distance from the base station.

\section{References}

[1] Green Solutions for Telecom Towers: Part I @ 2013 Intelligent Energy Limited.

[2] S. S. Krishnan, N. Balasubramanian, and A. M. Ramakrishnan, "Energy consumption and $\mathrm{CO}_{2}$ emission in India," Fourth International Conference on Infrastructure Systems and Services Next Generation Infrastructure Systems. Virginia, 2011.

[3] Telecom Regulatory Act of India, "Consultation paper on green telecommunications," 2011.

[4] L. M. Correia, D. Zeller, O. Blume, D. Ferling, and L.V. der Perre, "Challenges and enabling technologies for energy aware mobile radio networks," IEEE Communications Magazine, vol. 48, no. 11, pp. 62-72, 2010.

[5] Y. Chen, S. Zhang and S. Xu, "Fundamental trade-offs on green wireless networks," IEEE Communications Magazine, vol. 49, no. 6, pp. 30-37, 2011.

[6] J. Feng, C. Chang, S. Sayilir, Y. Lu, B. Jung, D. Peroulis and Y. C. $\mathrm{Hu}$, "Energy-efficient transmission for beamforming in wireless sensor networks," in Proc. IEEE Communication Society Conference on Sensor Mesh and Ad Hoc Communication and Networks (SECON), pp.1-9, 2010.

[7] B. Zayen, M. Haddad, A. Hayar and G. E. Oien, "Binary power allocation for cognitive radio networks with centralized and distributed user selection strategies," Physical Communication Journal, vol.1, no.3, pp. 183-193, 2008. 
[8] A. Gjendemsjø, D. G. Geir, E. Qien and S. G. Kiani, "Binary power control for sum rate maximization over multiple interfering links," IEEE Transactions on Wireless Communications, vol.7, no.8, 2008.

[9] S. G. Kiani, G. E. Oien and D. Gesbert, "Maximizing multicell capacity using distributed power allocation and scheduling," Proc. IEEE Wireless Communications and Networking Conference, 2007 (WCNC2007), pp. 1690-1694, 2007.

[10] Y. Zhao, S. G. Haggman, "BER analysis of OFDM communication systems with intercarrier interference," in Proc. International Conference on Communication Technology (ICCT'98), pp.1-5, 1998.

[11] Xu,Yongjun and Xiaohui Zhao. "Robust Probabilistic Distributed Power Control Algorithm for Underlay Cognitive Radio Networks under Channel Uncertainties."Wireless Personal Communications (2014): 1-16

[12] Pantazis, N.A, Vergados D.D., "A survey on power control issues in wireless sensor networks," Communications Surveys \& Tutorials, IEEE, vol.9, no.4, pp.86, 107, 2007.
[13] Xi Huang, X.Ge, Y.Wang and F. Li, "Energy efficient binary power control with bit error rate constraints in MIMO-OFDM wireless communication system," Vehicular Technology Conference (VTC Fall), 2012 IEEE, vol.1, no.5, pp. 3-6, 2012.

[14] Y.Wang, X. Ge, C.Cao, X. Huang and F. Y. Li, "Modeling and performance analysis of energy efficiency binary power control in MIMO-OFDM wireless communication systems," International Journal of Distributed Sensor Networks, vol.10, 2011.

[15] Z. Zhihua, X. He, and W. Jianhua, "Average power control algorithm with dynamic channel assignment for TDD-CDMA systems," Proceedings of the 2008 International Conference on Advanced Infocomm Technology, Shenzhen, China, July 2008.

[16] E.V.Belmega, S.Lasaulce and M.Debbah, "A survey on energyefficient communications," Personal, Indoor and Mobile Radio Communications Workshops (PIMRC Workshops), 2010 IEEE 21st International Symposium on, vol.289, no.294, pp.26-30, 2010 